\title{
Entrenamiento basado en simulación de eventos críticos para equipos de anestesiología: evaluación de habilidades no técnicas
}

\author{
Simulated crisis training for anesthesia teams: assessment of non-technical skills \\ Nicolás García,* Alejandro Delfino, * María Bravo, ${ }^{*}$ \\ Rodrigo Montaña,* Fernando Altermatt,* Marcia Corvetto*
}

Palabras clave:

Simulación, anestesiología, entrenamiento, eventos críticos.

Key words:

Simulation, anesthesiology, training, critical events.

* División de

Anestesiología, Facultad de Medicina, Pontificia Universidad Católica de Chile.

Recibido: 21/02/2019 Aceptado: 15/03/2019

\section{RESUMEN}

Introducción: El entrenamiento basado en la simulación para eventos críticos en anestesia es apropiado y factible. Sin embargo, la medición de la adquisición de habilidades y las mejoras en los resultados obtenidos después del entrenamiento de crisis basado en la simulación apenas se han evaluado. Objetivo: Determinar si el entrenamiento simulado de crisis mejora las habilidades no técnicas de los equipos de anestesia. Como objetivo secundario, se evaluó a los dos meses la autopercepción de los participantes. Métodos: Estudio de cohorte observacional prospectivo. Se desarrolló un curso de manejo de recursos en crisis de 2 días para equipos de anestesia, con el objetivo de mejorar las competencias de los participantes en las habilidades de manejo de recursos de crisis de anestesia. Los videos de todos los desempeños fueron revisados por dos evaluadores independientes y ciegos. Ellos calificaron las habilidades no técnicas de los participantes utilizando la escala ANTS (Anesthetist's Non-Technical Skills). Resultados: Todos los participantes finalizaron con éxito el curso. Varios aspectos de él fueron altamente calificados en la encuesta de satisfacción. Las puntuaciones promedio de los cuestionarios que evaluaron el conocimiento mejoraron del 54 al 81\%. No hubo mejoría en el puntaje total promedio de ANTS de los participantes, entre su primer y último escenario. La tasa de respuesta de una encuesta realizada dos meses después fue del $85 \%$ (17 de 20). Todos estuvieron de acuerdo en que mejoró su capacidad para manejar emergencias anestésicas. Conclusiones: La exposición a seis escenarios simulados de crisis en anestesia no mejora las habilidades no técnicas de los participantes, a pesar de una alta percepción de mejora en su práctica clínica. Investigaciones futuras deberían estar centradas en la evaluación de poblaciones de mayor tamaño y en el mantenimiento de las habilidades potencialmente adquiridas.

\section{ABSTRACT}

Background: Simulation-based training for anesthesia critical events is appropriate and feasible. However, measures of the acquisition of skills and outcome improvements after simulation-based crisis training for anesthesia teams have been scarcely assessed. Objective: to determine whether simulated anesthesia crisis training for anesthesia teams, improves nontechnical skills. As secondary outcome, a two months later survey was done aiming to collect self-perceived changes in their management of critical events. Methods: Prospective, observational cohort study. A 2-day anesthesia crisis resource management course was developed for anesthesia teams, with the objective of improving participant's proficiency in Anesthesia Crisis Resource Management (ACRM) skills. Videotapes of all performances were reviewed by two blinded independent raters. They rated participant's nontechnical skills using the Anesthetist's Non-Technical Skills (ANTS) score system. Results: All participants successfully finished the course. Several aspects of it were highly rated in the satisfaction survey. Average scores from questionnaires assessing knowledge improved from $54 \%$ to $81 \%$. There was no improvement in the mean total ANTS score of participants, between their first and last scenario. The response rate of the two months later survey was $85 \%$ (17 of 20). All of them agreed that it improved their ability to manage anesthetic emergencies. Conclusions: Exposure to six simulated anesthesia crises does not improve participant's nontechnical skills, despite a high perception of improvement in their clinical practice. Further research should be done in order to evaluate larger populations and to assess the maintenance of the potential acquired skills.

\section{INTRODUCCIÓN}

$\mathrm{E}^{\prime}$ entrenamiento de manejo de recursos en crisis en anestesiología (ACRM) se ha definido como la articulación de los principios del comportamiento individual y de grupo en situaciones ordinarias y de crisis que se centra en las habilidades de toma de decisiones dinámicas, el comportamiento interpersonal y el manejo del trabajo en equipo. ${ }^{1}$ Gaba comenzó en 1989 a desarrollar un plan de estudios basado en la simulación a partir del manejo de 
recursos en crisis (CRM) utilizado en la aviación y sus principios claves. ${ }^{2}$

Las habilidades no técnicas (NTS) se definen como aquellas cognitivas, sociales y de recursos personales que complementan las habilidades técnicas y que contribuyen a un rendimiento seguro y eficiente de una tarea determinada. ${ }^{3}$ Para el entrenamiento de estas habilidades se ha decidido adoptar un enfoque de manejo de recursos en crisis (CRM) mediante la utilización de sesiones de clases y simulaciones. ${ }^{4}$ Hoy en día, el desarrollo de esta amplia gama de marcadores conductuales desempeña un papel central en la buena práctica anestésica. ${ }^{5}$ Existe evidencia de que las NTS tienen una significativa correlación con las habilidades técnicas ${ }^{6}$ y que son un método útil para explicar las diferencias de rendimiento. ${ }^{7}$

Con el objetivo de evaluar las habilidades no técnicas, se ha desarrollado una serie de herramientas. ${ }^{8}$ La escala ANTS (Anaesthetists' Non-Technical Skills) fue elaborada en el año 2013 por Fletcher, con un satisfactorio nivel de validez, fiabilidad y aplicabilidad en el contexto experimental. ${ }^{9}$ Su propósito es describir y dar ejemplos de comportamientos que indiquen la presencia o ausencia de habilidades y actitudes definidas en el contexto del trabajo en equipo en el pabellón. A través de cuatro categorías (gestión, trabajo en equipo, consciencia de situación y toma de decisiones) y 13 elementos, este sistema nos da un puntaje final del desempeño evaluado.

En este contexto, como ya se definió, la capacitación de manejo de recursos en crisis en anestesiología basada en la simulación ha demostrado ser adecuada y factible. ${ }^{10}$ Un estudio de CRM que evaluó la capacitación de 499 anestesistas formados proporcionó evidencia que respalda que este tipo de metodología debería ser fomentado más ampliamente. ${ }^{11}$ Sin embargo, la medición de la adquisición de habilidades y las mejoras en los resultados después del entrenamiento de crisis basado en la simulación para los anestesiólogos apenas han sido evaluadas.

El objetivo principal de este estudio fue determinar si el entrenamiento simulado de eventos críticos para equipos de anestesia mejora las habilidades no técnicas de los participantes. Como objetivo secundario, se realizó una encuesta dos meses después del curso con el objetivo de recopilar cambios autopercibi- dos en el manejo de los eventos críticos en la práctica clínica.

\section{MATERIAL Y MÉTODOS}

Grupo estudiado. Tras la aprobación del Comité de Ética Institucional de la Facultad de Medicina de la Pontificia Universidad Católica de Chile y el consentimiento informado por escrito, 20 participantes de un curso institucional de manejo de recursos en crisis fueron enrolados para este estudio de cohorte, prospectivo, observacional. El grupo incluyó cuatro residentes de anestesiología, ocho enfermeros(as) de pabellón y ocho anestesiólogos formados, de los cuales la mayoría tenía experiencia previa en la participación en escenarios de simulación clínica.

Diseño del estudio e intervención. Se desarrolló un curso de manejo de recursos para crisis en anestesiología de dos días de duración para equipos de anestesia del pabellón de nuestra institución, con el objetivo de mejorar el dominio de los participantes en las habilidades de manejo de recursos de crisis de anestesia (ACRM, en inglés). Una semana antes del curso, se distribuyó literatura relacionada con cada uno de los escenarios de simulación.

Antes de las sesiones de simulación, se realizó una orientación de 30 minutos para todos los participantes con el objetivo de explicarles los principios de $\mathrm{ACRM}^{2}$ y las categorías y elementos incluidos en la escala ANTS. ${ }^{9}$ Cada equipo fue conformado por dos anestesiólogos, un residente de anestesiología y dos enfermeros(as).

Durante el curso, cada equipo fue expuesto a seis diferentes escenarios simulados utilizando un simulador de paciente de alta fidelidad. Los escenarios de eventos críticos fueron cesárea de emergencia, hipertermia maligna, manejo de vía aérea difícil, anafilaxia, laringoespasmo en paciente pediátrico e intoxicación sistémica por anestésicos locales. Estos seis escenarios son parte del programa de entrenamiento basado en la simulación para los residentes de anestesiología de la Pontificia Universidad Católica de Chile. ${ }^{12}$

Todos los escenarios fueron grabados en video. Posteriormente, en cada uno de ellos, un instructor entrenado guió la sesión informativa (debriefing), centrado en tanto el rendimiento de las habilidades técnicas como de las no téc- 


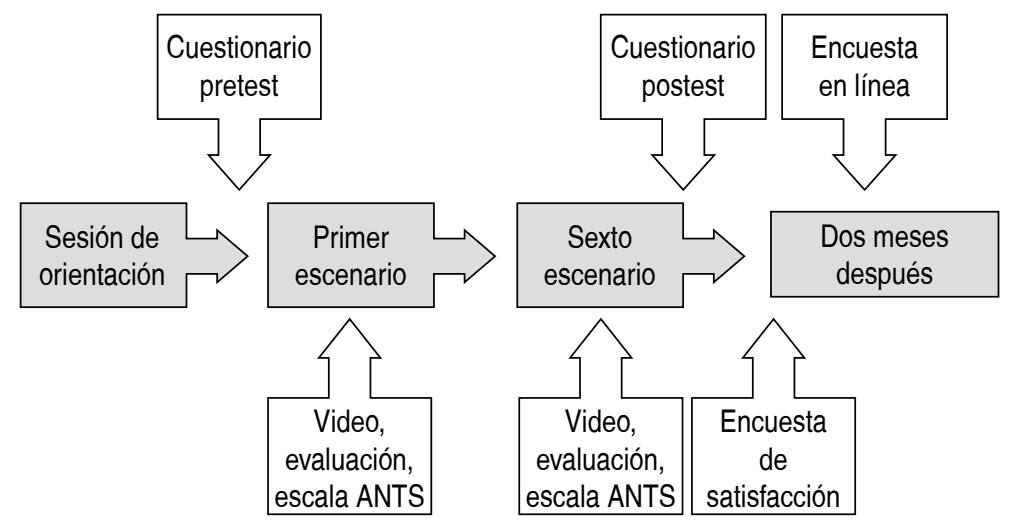

Figura 1: Diagrama de métodos.

nicas. Todos los informes fueron estructurados según el abordaje descrito por Rudolph. ${ }^{13}$

Instrumentos de medición y resultados. La evaluación de las habilidades no técnicas fue realizada a través de la escala $\mathrm{ANTS}^{9}$ aplicada al líder del equipo durante el primer y sexto escenario. Estas evaluaciones fueron llevadas a cabo por dos evaluadores independientes y ciegos entrenados en el uso de este instrumento de medición.

Todos los participantes completaron los cuestionarios, previa y posteriormente a cada escenario para evaluar su conocimiento respecto al tema. El cuestionario consistió en 35 preguntas de opción múltiple sobre los temas ya mencionados, sacadas de un grupo de preguntas diseñadas para nuestro programa de postgrado.

Una encuesta fue distribuida inmediatamente después de la finalización del curso para valorar el grado de satisfacción respecto a los escenarios y las sesiones informativas. Las respuestas obtenidas fueron procesadas en forma anónima.

Al final, se realizó una encuesta dos meses después con el objetivo de recolectar información de los cambios autopercibidos en el manejo de eventos críticos. La encuesta se diseñó utilizando la plataforma en línea disponible en el mercado Surveymonkey ${ }^{\circledR}$. Consistió en preguntas estructuradas que evaluaron múltiples aspectos con respecto al cambio autopercibido en el manejo de recursos en crisis en eventos críticos o difíciles de su práctica clínica. ${ }^{10}$ Las respuestas fueron procesadas anónimamente. La figura 1 muestra el flujo temporal de todas las evaluaciones.
Análisis estadístico. Los datos demográficos se expresaron como medias y desviación estándar ( \pm DE). El análisis de los puntajes de la escala ANTS se realizó con una prueba $t$ pareada para ambos grupos y categorías. El nivel de acuerdo entre los dos evaluadores se efectuó mediante una prueba $\kappa$ de Cohen. Los datos se analizaron con R Core Team (2013). R: Un lenguaje y entorno para la informática estadística. R Foundation for Statistical Computing, Viena, Austria. URL http://www.R-project.org/. Se consideró un valor $p$ menor de 0.05 como estadísticamente significativo.

\section{RESULTADOS}

Todos los participantes terminaron con éxito el curso. La puntuación total de la categoría ANTS asignada por cada evaluador para cada líder, con un rango entre 0 y 16 , se muestra en la tabla 1, sin diferencias entre el primero y el sexto escenario. La confiabilidad entre los evaluadores para la puntuación total de la categoría ANTS evaluada mediante la prueba $\kappa$ de Cohen fue $\mathrm{W}=0.432$ (valor $\mathrm{p}=0.763$ ).

Los puntajes para cada categoría evaluada por ANTS, con un mínimo de 0 y un máximo de 4 , se muestran en la tabla 2 , sin diferencias entre la primera y la sexta etapa.

Con respecto a los cuestionarios previos y posteriores al curso, que evalúan la adquisición de conocimiento de los participantes, sus puntajes promedio mejoraron de 54 a $81 \%$ (valor de $p<0.005)$.

Varios aspectos del curso obtuvieron una alta calificación en la encuesta de satisfacción. Todos los participantes calificaron el curso

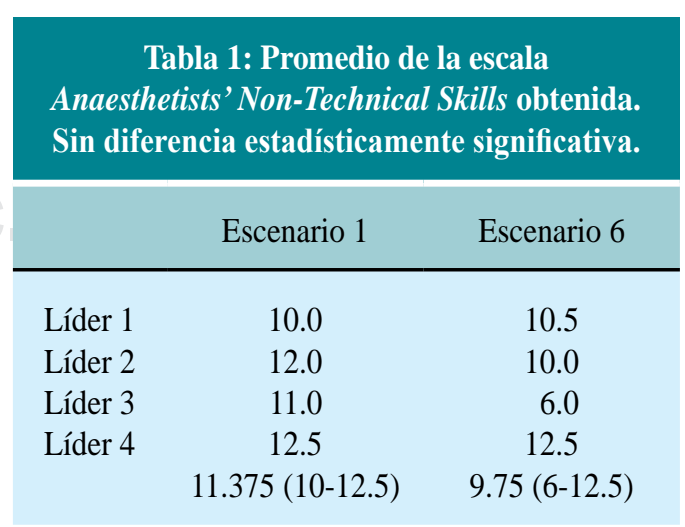


Tabla 2: Puntuación promedio (rango) por categoría de la escala Anaesthetists' Non-Technical Skills. Sin diferencias estadísticamente significativas.

\begin{tabular}{lrr} 
& Escenario 1 & Escenario 6 \\
\hline Administración de tareas & $2.75(2-4)$ & $2.25(1-3)$ \\
Trabajo en equipo & $2.875(1-4)$ & $2.5(2-4)$ \\
Consciencia de la situación & $3(1-3)$ & $2.25(1-4)$ \\
Toma de decisiones & $2.75(2-4)$ & $2.75(2-4)$
\end{tabular}

como excelente, concordaron en que los escenarios eran consistentes con los objetivos y que las sesiones informativas fueron conducidas de manera adecuada. El 95\% de los participantes estuvo de acuerdo en que los escenarios eran realistas. Es de destacar que $90 \%$ de los participantes estuvieron de acuerdo en que las actividades les causaron ansiedad y/o nerviosismo (Figura 2).

La tasa de respuesta de la encuesta de dos meses fue de $85 \%$ (17 de 20). Todos estuvieron de acuerdo en que el curso era relevante para su práctica clínica y que mejoraba su capacidad para manejar emergencias anestésicas. El 82\% estuvo de acuerdo en que el curso cambió su práctica clínica. Específicamente, $76 \%$ de los participantes estuvo expuesto a eventos críticos desde que tomaron el curso, informando una mejora en varios aspectos de su gestión durante las crisis, como se muestra en la figura 3.

\section{DISCUSIÓN}

El entrenamiento basado en la simulación con seis escenarios de alta fidelidad para el manejo de crisis no mejoró las habilidades no técnicas de los anestesiólogos, utilizando la escala ANTS. Pese a ello, se observó un aumento significativo del conocimiento y una alta percepción de mejoría en la práctica clínica.

Varias razones pueden explicar estos resultados. En primer lugar, hay evidencia que permite afirmar que las habilidades no técnicas están estrechamente relacionadas con las habilidades técnicas. ${ }^{6}$ En este contexto, es muy difícil encontrar diferencias después de una capacitación simulada en participantes con alto conocimiento teórico previo (anestesió- logos formados). Estudios previos realizados con residentes demostraron una mejora en los puntajes de ANTS después de la educación basada en la simulación. ${ }^{14-16}$ En este sentido, sería importante destacar que todos los anestesiólogos incluidos en este estudio se desempeñan en un hospital universitario, en un contexto de permanente docencia a residentes y alumnos de pregrado, por lo que no sólo están expuestos a las situaciones críticas diarias de su práctica anestésica, sino también deben ser capaces de transmitir y enseñar a sus residentes estrategias en el manejo de crisis y retroalimentar el desempeño del equipo tras la ocurrencia de éstas. Por lo tanto, otra posible causa de los resultados obtenidos podría ser la naturaleza universitaria del contexto en el que se desarrolló este estudio.

En segundo lugar, el diseño del estudio podría jugar un importante rol. En relación con el orden de los seis escenarios realizados, los primeros escenarios evaluados fueron toxicidad sistémica local anestésica y vía aérea difícil. Ambos son relativamente fáciles de manejar por un anestesiólogo general, lo que puede explicar los altos puntajes iniciales. Terminamos con una cesárea de emergencia y un laringoespasmo pediátrico, escenarios menos frecuen-

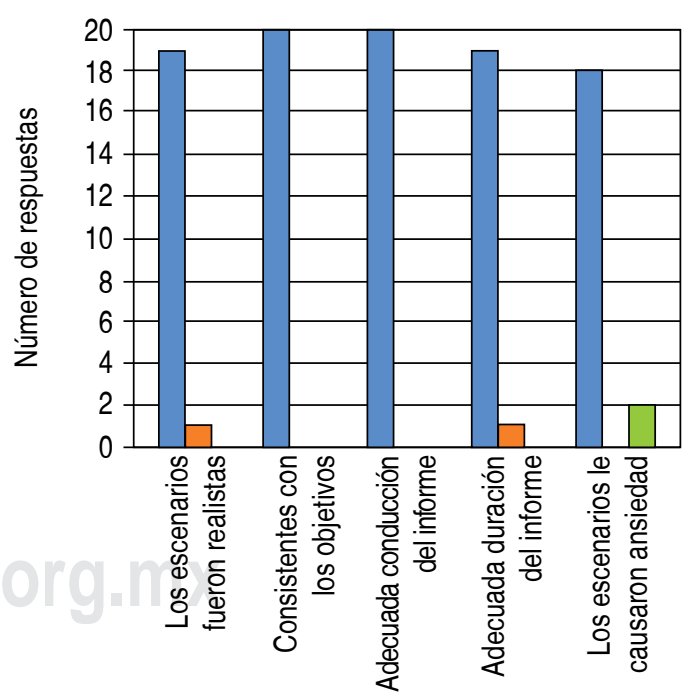

$\square$ Muy de acuerdo y de acuerdo

$\square$ Neutral

$\square$ Muy en desacuerdo y en desacuerdo

Figura 2: Resultados de la encuesta de satisfacción. 


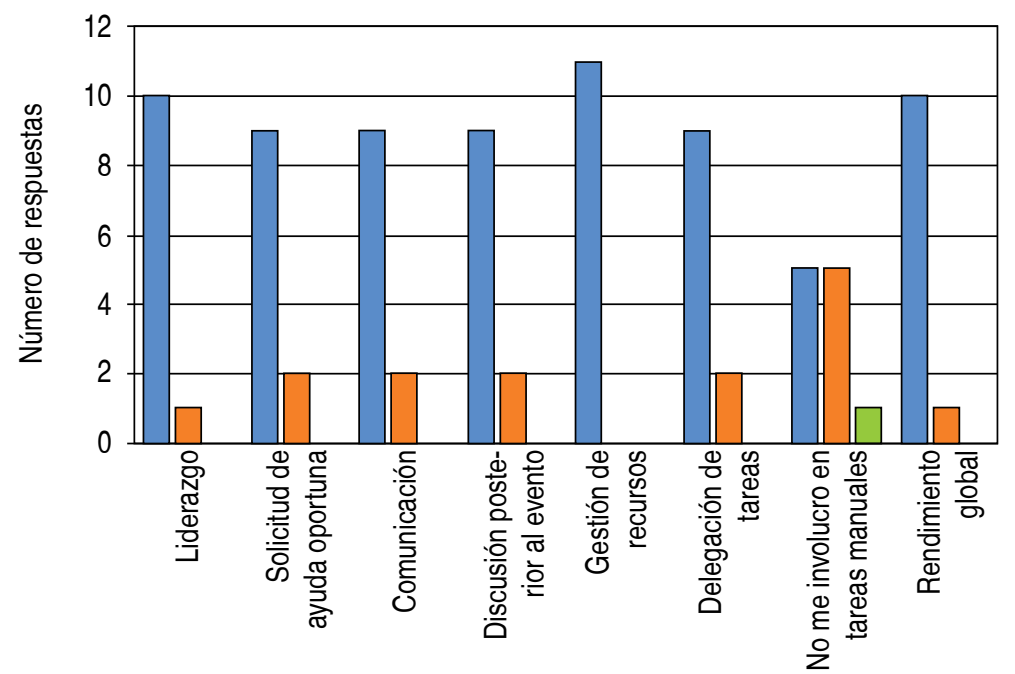

Significativamente mejorado y mejorado Significativamente deteriorado y deteriorado Neutral

Figura 3: Resultados de los cambios autopercibidos en eventos críticos reales.

tes, que requieren anestesistas especializados para manejar una crisis obstétrica y pediátrica, respectivamente.

En tercer lugar, nuestro plan de estudios basado en simulación se encuentra orientado a residentes, por lo que tiene la intención de entrenar y mejorar tanto habilidades técnicas como no técnicas, a fin de proporcionar una educación equilibrada. ${ }^{12}$ Esto podría llevar a un entrenamiento no suficientemente intenso para lograr una mejora significativa de habilidades no técnicas.

Por otra parte, desde el punto de vista estadístico, la razón de no encontrar diferencias significativas podría ser secundaria al hecho de que este estudio se insertó en un curso de manejo de recursos en crisis. Se puede correr el riesgo de carecer de poder suficiente para encontrar diferencias significativas dado el número de participantes. Dicho de otra forma, puede existir, de manera eventual, riesgo de error beta.

Un estudio previo con una metodología similar no demostró ninguna mejora apreciable en las habilidades no técnicas de anestesiólogos utilizando la escala ANTS como instrumento de medición. ${ }^{8}$ Estos resultados son consistentes con un metaanálisis reciente que sugiere que la medición de las habilidades no técnicas varían con amplitud entre los estudios y que el entre- namiento de dichas habilidades podría haber sido insuficientemente intenso en estos estudios.

Nuestro trabajo cuenta con varias limitaciones. Algunas de ellas ya han sido mencionadas, tales como el bajo número de participantes para evaluar, el uso de seis escenarios diferentes durante el entrenamiento, un orden de los casos que podría tener un efecto sobre la capacidad de encontrar diferencias entre el primer y sexto escenario y el posible impacto de la capacitación técnica en detrimento de las habilidades no técnicas. La confiabilidad entre evaluadores de nuestros dos evaluadores fue muy baja, a pesar de que han sido entrenados en el sistema ANTS. Sin embargo, los estudios previos que utilizaron ANTS han mostrado bajos puntajes de confiabilidad entre evaluadores. En un estudio realizado por Yee, ${ }^{17}$ el coeficiente de correlación interclase (ICC) entre dos observadores fue 0.53 en el nivel de categorías y 0.5 en el nivel de elementos. De manera similar, Savoldelli reportó un ICC en el nivel de categorías de 0.58 , también con dos observadores. ${ }^{14}$ Finalmente, en un estudio realizado por Graham, ${ }^{18} 26$ anestesiólogos fueron entrenados para usar el sistema de puntuación ANTS mediante un curso de ocho horas de duración. El ICC para cada elemento varió de 0.1 a 0.6; sin embargo, no lograron un nivel aceptable de correlación de 0.7 .

\section{CONCLUSIONES}

Teniendo en cuenta las consideraciones mencionadas, nuestros resultados son consistentes con la literatura previa en un contexto donde es difícil encontrar mejoras en las habilidades no técnicas, ya sea por las limitaciones en el rendimiento de las escalas de medición actualmente disponibles para evaluar este tipo de habilidades o por la por la población en la que éstas fueron aplicadas en este estudio. Desde nuestro punto de vista, fallamos porque nuestros participantes ya eran anestesistas entrenados. Probablemente, el impacto del entrenamiento basado en simulación en el manejo de recursos en crisis sobre la mejora de las habilidades no técnicas tenga un impacto diferente de acuerdo a la población estudiada (por ejemplo, anestesiólogos sin experiencia en simulación clínica, anestesiólogos que trabajen en hospitales no universitarios y residentes de 
especialidad). Proponemos un nuevo estudio para corregir los errores metodológicos ya planteados, sobre todo centrados en el cálculo de una potencia adecuada, e intentar motivar a otros grupos para hacer estudios multicéntricos que nos permitan demostrar las capacidades de esta importante herramienta de aprendizaje.

\section{REFERENCIAS}

1. Gaba DM. Crisis resource management and teamwork training in anaesthesia. Br J Anaesth. 2010; 105: 3-6.

2. Gaba DM, Howard SK, Fish KJ, Smith BE, Sowb YA. Simulation-based training in anesthesia crisis resource management (ACRM): a decade of experience. Simul Gaming. 2001; 32: 175-193.

3. Flin R, Patey R, Glavin R, Maran N. Anaesthetists' non-technical skills. Br J Anaesth. 2010; 105: 38-44.

4. Flin R, Maran N. Identifying and training non-technical skills for teams in acute medicine. Qual Saf Health Care. 2004; 13: i80-84.

5. Fletcher GC, McGeorge P, Flin RH, Glavin RJ, Maran NJ. The role of non-technical skills in anaesthesia: a review of current literature. Br J Anaesth. 2002; 88: 418-429.

6. Riem N, Boet S, Bould MD, Tavares W, Naik VN. Do technical skills correlate with non-technical skills in crisis resource management: a simulation study. $\mathrm{Br}$ J Anaesth. 2012; 109: 723-728.

7. Carthey J, de Leval MR, Wright DJ, Farewell VT, Reason JT. Behavioural markers of surgical excellence. Safety Science. 2003; 41: 409-425.

8. Morgan PJ, Kurrek MM, Bertram S, LeBlanc V, Przybyszewski T. Nontechnical skills assessment after simulation-based continuing medical education. Simul Healthc. 2011; 6: 255-259.

9. Fletcher G, Flin R, McGeorge P, Glavin R, Maran N, Patey R. Anaesthetists' non-technical skills (ANTS): evaluation of a behavioural marker system. $\mathrm{Br}$ J Anaesth. 2003; 90: 580-588.

10. Blum RH, Raemer DB, Carroll JS, Sunder N, Felstein DM, Cooper JB. Crisis resource management training for an anaesthesia faculty: a new approach to continuing education. Med Educ. 2004; 38: 45-55.
11. Weller J, Morris R, Watterson L, Garden A, Flanagan B, Robinson B, et al. Effective management of anaesthetic crises: development and evaluation of a collegeaccredited simulation-based course for anaesthesia education in Australia and New Zealand. Simul Healthc. 2006; 1: 209-214.

12. Corvetto MA, Bravo MP, Montana RA, Altermatt $F R$, Delfino AE. Bringing clinical simulation into an anesthesia residency training program in a university hospital. Participants' acceptability assessment. Rev Esp Anestesiol Reanim. 2013; 60: 320-326.

13. Rudolph JW, Simon R, Dufresne R, et al. There's no such thing as "non judgmental" debriefing: A theory and method for debriefing with good judgment. Simul Healthc. 2006; 1: 49-55.

14. Savoldelli GL, Naik VN, Park J, Joo HS, Chow R, Hamstra SJ. Value of debriefing during simulated crisis management: oral versus video-assisted oral feedback. Anesthesiology. 2006; 105: 279-285.

15. Welke TM, LeBlanc VR, Savoldelli GL, Joo HS, Chandra DB, Crabtree NA, et al. Personalized oral debriefing versus standardized multimedia instruction after patient crisis simulation. Anesth Analg. 2009; 109: 183-189.

16. Boet S, Bould MD, Bruppacher HR, Desjardins F, Chandra DB, Naik VN. Looking in the mirror: selfdebriefing versus instructor debriefing for simulated crises. Crit Care Med. 2011; 39: 1377-1381.

17. Yee B, Naik VN, Joo HS, Savoldelli GL, Chung DY, Houston PL, et al. Nontechnical skills in anesthesia crisis management with repeated exposure to simulationbased education. Anesthesiology. 2005; 103: 241-248.

18. Graham J, Hocking G, Giles E. Anaesthesia nontechnical skills: can anaesthetists be trained to reliably use this behavioural marker system in 1 day? $\mathrm{Br}$ J Anaesth. 2010; 104: 440-445.

\section{Correspondencia:}

Marcia A Corvetto

División de Anestesiología, Facultad de Medicina, Pontificia Universidad Católica de Chile. Marcoleta $377,4 .^{\circ}$ piso, Santiago Centro, Chile. Tel: (562) 2354 3270; Fax: (562) 26327620

E-mail: marciacorvetto@gmail.com 